\title{
Indexing and Reviewing: Concept and Its Practice
}

\author{
Akshatha Banadka \\ Research Scholar \\ Department of Life Sciences \\ Christ (Deemed to be University) \\ Bengaluru, Karnataka, India \\ akshatha.b@res.christuniversity.in
}

\begin{abstract}
Indexing is a crucial factor that helps journal to reach out a larger audience and achieve reputation by standing out from the rest that are crowding in publication space. This article helps gaining an understanding on the concept of indexing, its history, its practices, the major indexing agencies and the indexing parameters. The paper also discusses on the different review methods adopted by journals and the concept of open access journals.
\end{abstract}

Keywords - Indexing, Indexing Agencies, Indexing Parameters, Review Methods, Open Access Journal.

\section{Introduction}

"Words are powerful than some noises", says Billie Eilish and indexing is the one which helps a journal to make noise and stand out from the rest of the journals in the publishing space. Indexing helps a journal getting its publication indexed by one or more leading indexing agencies. Indexing is a systematic process of arranging the list of cited articles identified as 
source and each cited article in turn accompanied by a list of citing articles identified as reference. An indexed journal is considered to have a better quality and reputation as compared to non-indexed journals most of the times. However, sometimes an indexed journal need not necessarily have a better quality than a non-indexed journal. This is because most of the established journals refused to apply and undergo a long and tight process to be indexed.

\section{Methodology}

In the current article, a detailed investigation of the concept of indexing, its history, the different indexing agencies and indexing parameters has been made. The parameters set up by the major indexing agencies has been analysed. The different types of review methods followed by different journals has been studied to understand the review process. An in-depth study on open access journal with an emphasis on funding mechanism of open access journal has been made.

\section{History of indexing}

Everything has its own history so does the indexing. The concept of indexing was formed from the bits and pieces of the information that has been accumulated over the years. The history of indexing dates back to $7^{\text {th }}$ Century where Indexing was used to list the Concordances to Bible. Indexing was also used for subject indexes to canonical law in the $11^{\text {th }}$ century. In, $12^{\text {th }}$ century, a form of citation index was found in Hebrew religious literature. However, the concept of indexing gained its prominence in the fifteenth century with the contrivance of printing. The first printed book with indexes was released in 1460's. Later, in the $16^{\text {th }}$ century, i.e., in 1544 , the first printed Biblical concordance was published. The first index to the English language in $18^{\text {th }}$ century was "A dictionary of the English language" by Samuel Johnson released in 1755. 
In the $19^{\text {th }}$ century, the legal citation indexes were developed and were made popular by citators such as Shepard's citations (1873). It is the oldest major citation index which provided a tool for searching legal decision and is still in existence. In 1877, The Index Society was formed in London for creating a general index of universal literature. With these, in 1960, the first citation index for papers was published in academic journals which introduced by Eugene Garfield's Institute for Scientific Information (ISI). In the year 1997, "Cite Seer" did the first automated citation indexing.

Indexing got its notability with the advent of Computers. The computerization had a positive impact and helped in the process of indexing by organizing and recording the huge amount of database. However, it also has its own drawbacks such as the quality is compromised. The information available is not subject to any kind of screening or quality check. Currently, Indexing is one of the important aspects while considering the prestige of a journal.

\section{Indexing agencies}

For a journal to be indexed, it has to pass through a review process of certain requirements done by a journal indexer. There are many indexing agencies available at present. While some indexing agencies such as Scopus are provided by publishers, others such as PubMed are affiliated with institutions like National institute of health (NIH) Irrespective of the indexing agency, for inclusion of the journal in the database (indexing agency) the journal has to formally apply and fulfill all the parameters set by the indexing agency

The indexing agencies can only index titles or only the abstract and/or references or full articles. Once a journal is indexed by the indexing agency, the journal is immediately made available to all users of that database. 
The major indexing agencies are:

\subsection{Web of Science}

Web of Science is an interdisciplinary database that covers all scientific areas. It is one of the most reputed database that covers best international and regional journals, proceedings, books and patents that are only in English language. The articles in the database are entered in a uniform fashion with the details such as the name of the author, the title of the paper and the journal and reliably sorted out latest name. Thus, the articles of interest can be easily retrieved by the reader.

The Web of Science has three flagship Citation Indexes and they are:

- the Science Citation Index Expanded (SCIE) earlier known as SCI (Science Citation Index),

- the Social Sciences Citation Index (SSCI).

- $\quad$ the Arts \& Humanities Citation Index (AHCI).

- A new edition, Emerging Sources Citation Index.

Approximately 7,000+ academic and research institutions, funding organizations, publishing organizations and national governments are maintained by Web of science worldwide.

\subsection{Scopus}

Scopus is one of the major indexing databases owned by Elsevier that contains abstracts and citations for academic journal articles covering approximately 21,000 titles. The peer reviewed scientific journals, books and conference proceedings in the fields of science, technology, medicine, social sciences, and arts and humanities are covered by Scopus. The research can be tracked, analyzed and visualized by smart tools featured by Scopus. This bibliographic database ensures that the research in its journals becomes increasingly global, 
interdisciplinary and concerted. Scopus also gives the H-Index and citation impact of the journal.

\section{3. DBLP (Digital Bibliography \& Library Project)}

DBLP computer science bibliography is a collaboration of the University of Trier and Schloss Dagstuhl which specifically gives an information on major computer science journals and proceedings. DBLP mainly focuses on international publications in English. DBLP prioritizes the inclusion of publication venue for the computer science community based on their scientific merit and regular publishing history as there are no resources that cover this venue exists. For the journals to be indexed by DBLP, the journal has to fulfil the minimum standards set by the DBLP advisory board has defined a set of minimum standards that any publication series should meet in order to be indexed by DBLP.

\subsection{MEDLINE, PubMed, PubMed Central}

MEDLINE is the journal database of national library of medicine (NLM). It was established in the 1960's and provides nearly 26 million citations of medical and life science related journal articles since 1946 and indexes approximately 5,200 journals.

In 1996, PubMed was started. It has more than 30 million citations which include the MEDLINE database along with the in-process citations, citations for the out-of-scope articles, citations that precede articles published in a journal before it was MEDLINE indexed, citations of those that are submitted ahead the print, citations articles submitted published by NIH and PubMed Central. It also includes citations of the books, and book chapters in the NCBI Bookshelf.

PubMed Central is a digital repository maintained by the National Center for Biotechnology Information (NCBI) at the National Library of Medicine (NLM), which was 
launched in 2000. The database archives abstract and full-text articles freely accessible to the public. It is database with approximately 1600 journals that mainly focuses on biomedical and clinical journals. The repository contains citations, abstracts and full text articles in the fields of medicine and healthcare systems from MEDLINE.

\subsection{CrossRef}

CrossRef, an official Digital Object Identifier (DOI) Registration Agency of the International DOI Foundation established in early 2000 is run by the Publishers International Linking Association Inc. (PILA). It is a non-profit association with $60 \%$ non-profit organizations that has 3237 publishers and facilitates the linking of cross-publisher citation among the publishers in online academic journals. CrossRef has been providing a reference linking services for 44 million items which includes journals, books, book chapters and proceedings serving approximately 1600 libraries since its establishment.

\subsection{Google Scholar}

Google Scholar is a Google's search engine which permits the searches of abstracts, articles, journals, thesis, from academic publishers, repositories and universities etc. across various disciplines. It is a non-human curated database that enhances the global visibility and accessibility of the research content.

In conclusion, MEDLINE, PubMed Central, ISI, and Scopus are the four major indexing agencies as per Wolters Kluwer.

\section{Indexing parameters}

When an author publishes an article based on the number of the articles published by the author, it can be quantified. However, the quality of the journal and the articles published 
by the article can be measured using the indexing parameters which includes bibliometric indices. The quality check is done by two ways:

1. Examining the impact and influence of journals.

2. Determining an author's academic contribution with a defined set of values.

5.1 Examining the impact and influence of journals: This can be evaluated by calculating the quasi-quantitative indicators such as impact factor and eigen factor score of the journal

\subsubsection{Journal impact factor:}

Impact factor (IF) is published in the Journal citation report annually by Thomson Reuters. If of a journal in a year D is defined as the total number of citations of the articles that are published in a journal in D-1 and D-2 years divided by the total number of articles that are published in the same journal over the same two years D-1 and D-2. Recently a journal impact factor for five years given by Thomson Reuters has been in existence.

\subsubsection{Eigenfactor Score:}

The impact factor gives equal value on citations irrespective of the influence and quality of journal, to overcome this, Bergstom devised Eigenfactor score (ES), which gives higher values to journals with a good quality and influence. An iterative ranking algorithm system is employed to calculate the ES and it is calculated based on the frequency with which the journal is visited by an author and expressed in percentage. The ES is calculated every year for an interval of 5 years. 


\subsubsection{SCImago Journal Rank:}

SCImago Journal Rank is a computerized ranking system devised by SCImago research group using Scopus. It is a ranking system that uses an algorithm to assign a higher rank to quality journals with more number of citations. The SJR is published in SCImago Journal. 5.2 Determining an author's academic contribution with a defined set of values: This includes bibliometric indices that measures the quantity and quality of author's research output. Some of the most commonly used bibliometric indices are explained below:

\subsection{1 h index:}

It is the most sought-after bibliometric index which was introduced by Jorge E. Hirsch at the University of California in 2005. It is a metric that is calculated based on the number of citations and the number of publications by an author. The $\mathrm{h}$ index is defined as the $\mathrm{x}$ number of articles, each cited $\mathrm{x}$ number of times. It is a non-copyrighted index that can be used by anyone. However, $\mathrm{h}$ index has a disadvantage as it cannot be compared across different disciplines as H-index can higher in one discipline when compared to others and there is a problem of self-citation.

\subsection{2 m index:}

It is another metric that was introduced by Hirsch is calculated by dividing the $h$ index of the author divided by the number of years the author has been actively involved in publication since his first year of publication. Even though, the $\mathrm{m}$ index takes into account the research age of an author, it has the same drawbacks of $h$ index. 


\subsection{3 g index:}

To overcome the drawbacks of $\mathrm{h}$ index and $\mathrm{m}$ index, another metric called $\mathrm{g}$ index was introduced by Leo Egghe in 2006. It is measured based on the highest citations that are received by an author. The $\mathrm{g}$ index is defined as the highest $\mathrm{x}$ number of articles that have received at least $\mathrm{x}^{2}$ number of citations when arranged in decreasing order of their citations.

\subsection{4 e index:}

As a complementary bibliometric index to $\mathrm{h}$ index, another independent index called e index was devised by Zhang. While measuring the $\mathrm{h}$ index, any excess number of citations beyond the $\mathrm{h}$ number of citations is not taken into consideration. However, e index is the measurement of the square root of excess citations used in the $\mathrm{h}$ index

\subsection{5 i10 index:}

This bibliometric index was designed by Google scholar to gauge the publications. It is a facile straightforward metric that is measured by the total number of articles with at least 10 citations.

\subsection{6 $\mathrm{h}^{\mathrm{c}}$ index:}

The contemporary $\mathrm{h}$ index was proposed by Sidiopoulis which gives weightage to newer articles than the older ones thereby giving importance luminous young researchers who has made significant contribution with small number of article. The $h^{c}$ index is calculated by multiplying the number of citations with four and then dividing it by the number of years of publication. 


\section{Reviewing process}

"True intuitive expertise is learned from prolonged experience with good feedback on mistakes." as said by Daniel Kahneman. Reviewing process is a feedback that is given by the reviewers for the authors to improve on the quality of the article. Reviewing is the most important scientific evaluation undertaken by the journals and indexing agencies. Reviewing process is broadly classified as pre- publication and post publication review.

\section{Peer review}

Peer review is the most common reviewing process that assess the quality and validate the content of the article and in turn broadens the network with the research community. The peer review process was first conducted by a journal Philosophical Transactions of the Royal Society in the year 1618. Peer review process is broadly categorized into open review and closed review which includes single blind, double blind and triple blind review.

\subsection{Single blind review:}

This is the most popular type of peer review process. In the single review, the author will not know who the reviewer is but the reviewer knows who the author is. This method allows the reviewer to research on the previous work done by the author and also allows the reviewer to provide feedback without fear of criticism. However, the identity of author might influence the reviewer and sometimes use their anonymity to make discordant comments.

\subsection{Double blind review:}

In double blind review neither the author nor the reviewer knows each other. The double-blind review process minimizes the reviewer bias and conflict of interest. But because of the author anonymity the reviewer cannot research on the previous work done by the author. 


\subsection{Triple blind review:}

In this review process, neither the author nor reviewer know each other and the author is not known to the editor as well. The triple blind review process has reduced review bias as the article is anonymized at the stage of article submission. Just like double blind review process, as the author is anonymized, it is not possible to get the information on the previously done work by the author.

\subsection{Open review:}

It is the most transparent review process where the author and the reviewer know each other. However very less number of journals follow open review process. This peer review has many pros such as thorough reviewing as the reviewers are aware of their identity and this method prevents plagiarism. However, there are cons like conflict of interest and sometimes the identity of the author might influence the reviewer.

\section{Open access journal}

A journal which seeks to grant permission to access its article, thereby making it available online free of cost for the readers is called as an open access journal. This unrestricted access to the scientific information without financial and technical blockade provides a platform for the readers especially the authors for an expeditious propagation of recent developments in the research field. A publication can be made openly accessible either by green OA which involves self-archiving the article for the free public use or by gold OA by publishing it in an open access journal or in an open access repository. 
9. Conclusion

Indexing is a marker of journal quality which enhances the availability, readership and visibility of a journal. It will help the journal to reach out a larger number of readers thereby increasing the citation number of the article. The enhanced citation number in turn will have an impact on the journal's reputation as a reliable source of high-quality information. The reviewing process is an important criterion for publishing a quality article in a journal. In conclusion, this article helps the upcoming researches to understand the basic concept of indexing and reviewing. 


\section{References}

Andrews, MEDLINE, PubMed, and PubMed Central: How are they different?", UC Berkeley Library Update, 2018. [Online]. Available: https://update.lib.berkeley.edu/2013/09/03/medline-pubmed-and-pubmed-centralhow-are-they-different/. [Accessed: 11- May- 2020]

Balhara Yatan Pal Singh, Indexed journal: What does it mean?, Lung India, 29(2), p. 193, 2012

Chatterjee Sharmili Indexing: History and its usage, International Journal for Research in Engineering Application \& Management, 5(6),126-128, 2019

Choudhri A F., et al., Understanding Bibliometric Parameters and Analysis, RadioGraphics, vol 35 (3) 736-746, 2015

Dhammi IshKumar, Haq RU., What is indexing. Indian J Orthop. 50(2):115-116. 2016

Feroz Sharmila .,."Indexing - Meaning, History, Types, Agencies and Parameters", Journal of the Gujarat Research society, 21(3), 1-8, 2019

Garner Rebecca M, Hirsch Joshua A et al., Bibliometric indices: defining academic productivity and citation rates of researchers, departments and journals, Journal of NeuroInterventional Surgery, vol. 10, no. 2, pp. 102-106, 2017

Gokula. Nishitha, Research Publication- Indexing and Other Aspects, International Journal of English Language, Literature in Humanities, vol. 7, no. 9, pp. 501-514, 2019. [Accessed 10 May 2020]

Harzing Anne-Wil ., Metrics: h and g-index, Harzing.com, 2016. [Online]. Available: https://harzing.com/resources/publish-or-perish/tutorial/metrics/h-and-g-index. [Accessed: 12- May- 2020].

Joshi M.A, Bibliometric Indicators for Evaluating the Quality of Scientific Publications, The Journal of Contemporary Dental Practice, vol. 15, no. 2, pp. 258-262, 2014 
Kumar Shanu, How to Get Your Articles Indexed in PubMed: The Go-To Guide for Publishers, Typeset, 2020.

Levene et al., A bibliometric index based on the complete list of cited Publications.

Cybermetrics. 16(1), 1-2, 2013

LibGuides: Open Access Publishing: What is Open Access? Guides.library.cornell.edu, 2020. [Online]. Available: https://guides.library.cornell.edu/openaccess. [Accessed: 13May- 2020].

Maliakkal Deepthi Jose., Indexing: History, Concept and Practice, International Journal of Social Sciences, 8(4), 123-128, 2019.

McInerney James., H-Index, M-Index and google citations, James McInerney's Lab Website, 2011. [Online]. Available: http://mcinerneylab.com/research/h-index-m-index-andgoogle-citations/\#. [Accessed: 13- May- 2020].

MEDLINE, PubMed, and PMC (PubMed Central): How are they different?, Nlm.nih.gov, 2019. [Online]. Available: https://www.nlm.nih.gov/bsd/difference.html. [Accessed: 11- May- 2020].

Padula. Danielle, Journal Indexing: Core standards and why they matter, LSE Impact Blog, 2020

Rajagopalan Jayashree., "Journal indexing 101: Understanding the basics", Editage Insights, 2020. [Online]. Available: https://www.editage.com/insights/journal-indexing-101understanding-the-basics. [Accessed: 10- May- 2020].

Rose Divya., Indexing, reviewing and publishing - Being a public intellectual, International Journal of Advance Research, Ideas and Innovations in Technology, 5(5) 259-262, 2019

Shaik, Dr. Fahimuddin. 2016. An Importance of Indexing in Research Publications-A review article. 
Simkin John., A brief history of indexing, Anzsi.org. [Online]. Available:

https://www.anzsi.org/about-indexing/a-brief-history-of-indexing/

Types of Peer Review | Wiley, Authorservices.wiley.com. [Online]. Available:

https://authorservices.wiley.com/Reviewers/journal-reviewers/what-is-peer-

review/types-of-peer-review.html. [Accessed: 12- May- 2020].

What is Open Access? www.springer.com, 2020. [Online]. Available:

https://www.springer.com/gp/authors-editors/authorandreviewertutorials/openaccess/what-is-open-access/10286522. [Accessed: 13- May- 2020].

What is peer review? Elsevier.com. [Online]. Available:

https://www.elsevier.com/reviewers/what-is-peer-review. [Accessed: 12- May- 2020].

Wu Bo. et al., E-Index-A Bibliometric Index of Research Efficiency, in IEEE Access, 6, $51355-51364,2018$ 